\title{
Enhanced stability of nano-emulsified paclitaxel
}

\author{
Ju Young Lee ${ }^{1 \mathrm{a}}$, Da Yeon Kim ${ }^{1 \mathrm{a}}$, Gyeong Hae Kim ${ }^{1}$, Kkot Nim Kang ${ }^{1}$, Byoung Hyun Min ${ }^{1}$, Bong Lee ${ }^{2}$, \\ Jae Ho Kim ${ }^{1}$, Moon Suk Kim ${ }^{1}$
}

${ }^{1}$ Department of Molecular Science and Technology, Ajou University, Suwon, Korea;
${ }^{2}$ Department of Polymer Engineering, Pukyong National University, Busan, Korea.
Email: moonskim@ajou.ac.kr

Received 9 February 2011; revised 28 March 2011; accepted 4 April 2011.

\begin{abstract}
The main goal of this work was to develop an optimal self-microemulsifying paclitaxel prepared with PLGA and solubilizer such as tetraglycol, Cremophor ELP, and Labrasol. The prepared PTx-loaded SMES showed the size of the range of $80-130 \mathrm{~nm}$ by dynamic light scattering and a spherical shape by atomic force microscopy. In experiment of storage stability in deionized water (DW) or blood condition, PTx-loaded SMES showed good stability in DW and comparable stability in blood condition at $37^{\circ} \mathrm{C}$ for 7 days. In addition, PTx-loaded SMES showed a significant inhibitory effect on B16F10 melanoma proliferation. In conclusion, we confirmed that the formulations tried in this study could be used as administration form for animal trials of PTx.
\end{abstract}

Keywords: Self-Microemulsifying; Paclitaxel; Stability; Anti-Tumor Activity

\section{INTRODUCTION}

Paclitaxel (PTx), a major anticancer drug isolated from the bark of Taxus brevifolia, has significant activity in clinical trials against a variety of tumors such as breast cancer, advanced ovarian carcinoma, lung cancer, head and neck carcinoma [1,2]. PTx is a hydrophobic drug with poor aqueous solubility. To enhance its solubility and allow parenteral administration, PTx is currently formulated with solution of Cremophor ${ }^{\circledR}$ EL and ethanol as dosage-form of PTx (Taxol®) for clinical application $[3,4]$.

Recently, numerous investigations have been focused on the development of various PTx delivery systems such as liposomes, emulsions, micelles, microspheres, and polymeric nanoparticles [5-9]. Among these, selfmicroemulsifying systems (SMES) may be a promising way to load PTx in delivery system because it provides

${ }^{\mathrm{a}} \mathrm{Ju}$ Young Lee and Da Yeon Kim are equal first authors in this work. high concentration of PTx in the aqueous media system $[10,11]$. SMES are isotropic mixtures of oil, a surfactant, and possibly one or more hydrophilic solvents or cosurfactants, which form fine oil-in-water emulsions when exposed to aqueous media under condition of gentle agitation [12].

At present, many studies have highlighted the development of PTx-loaded SMES with optimal condition for blood circulation in effective concentration [13]. However, the major obstacle that limits the use of SMES is due to the physical and/or to the chemical instability by absorption of biological compounds such as protein during drug circulation time in blood after intravenous administration [14].

To improve stability of SMES, various formulations of SMES is needed that can extend PTx circulation time in blood. Our understanding is that the formulations of SMES could examine under the blood condition. Thus, the aim of this study was to examine various formulations of PTx-loaded SMES to increase systemic clearance through extending of the circulation time of PTx.

\section{MATERIALS AND METHODS}

\subsection{Materials}

Poly(d,1-lactide-co-glycolide) (PLGA, molecular weight, $8000,20,000$ and $90,000 \mathrm{~g} / \mathrm{mole}$ ) were purchased from Boerhinger Ingelheim (Ingelheim, Germany). Paclitaxel was purchased from Samyang Genex Co. (Seoul, Korea). Caprylocaproyl macrogol-8 glyceride (Labrasol ${ }^{\circledR}$ ) was obtained from Gattefosse (Westwood, NJ, USA). Cremophor ELP was purchased from BASF (Germany). Tetraglycol was purchased from Sigma Chemical Co. (St. Louis, MO, USA). All other chemicals were of reagent grade. Deionized water (DW) was prepared by a Milli-Q purification system from Millipore (Molsheim, France).

\subsection{Preparation of PTx-Loaded SMES}

A series of SMES was prepared in each of the various formulas with various ratios of PLGA, paclitaxel, solu- 
bilizer, surfactant, and cosurfactant. Briefly, PLGA and PTx were dissolved, respectively, by solubilizer such as tetraglycol in glass vials. Surfactant and cosurfactant were accurately weighed into glass vials. Then the components were mixed by gentle stirring and vortex mixing until PTx had perfectly dissolved. The mixture was stored at room temperature until used. Before using, PTx-loaded SMES was formed by contacting to aqueous phase of the prepared mixture.

\subsection{Size Analysis of PTx-Loaded SMES}

For particle size analysis, formulation $(50 \mu \mathrm{l})$ of PTxloaded SMES was diluted with DW to $50 \mathrm{ml}$ in a volumetric flask and gently mixed by inverting the flask. The droplet sizes of resultant emulsions were determined by dynamic light scattering (DLS, ELS-8000, Photal, Japan) at room temperature. The droplet size was individually measured for three PTx-loaded SMES samples and then calculated as average value. For atomic force microscopy (AFM), one drop of PTx-loaded SMES was transferred onto silicone wafer which washed with $\mathrm{MeOH}$. The wafer was quickly placed in liquid nitrogen, followed by the freeze-drying for 2 days. AFM measurements were carried out in the tapping mode with a Nanoscope IV instrument (Digital Instruments Inc.).

\subsection{Stability of PTx-Loaded SMES}

PTx-loaded SMES was prepared with DW or a solution of $0.9 \% \mathrm{NaCl}$ and $5 \%$ bovine serum albumin (Sigma, Germany) and individually placed in $10 \mathrm{ml}$ tube. The tube was constantly shaken at $100 \mathrm{rpm}$ and $37^{\circ} \mathrm{C}$ for 7 days. At the set time, the droplet size was individually measured for three PTx-loaded SMES samples and then calculated as average value.

\subsection{Cell Culture and Cytotoxicity Tests}

B16F10 melanoma cell line was obtained by KCLB (Korea Cell Line Bank) and cultured in MEM (Minimum Essential Medium, Gibco BRL, USA) supplemented with $10 \%$ fetal bovine serum (Gibco BRL, USA) The cells were seeded into $75 \mathrm{~cm}^{2}$ flasks, cultured and changed medium every 2 days. For cytotoxicity tests, B16F10 cell suspension $\left(2 \times 10^{4}\right.$ cells/well $)$ was seed in a 48 -well plate. The cells were incubated overnight to allow for cell attachment. Ptx $(1 \mu \mathrm{g})$ of SMES solution was added to each well for 7 days. Cell viability was determined by using water-soluble enzyme substrate MTT which was converted to purple water-insoluble product formazan accumulated in the cytoplasm of viable cells. Cell viability of each well performed individually and then calculated as average value. In brief, $100 \mu \mathrm{l}$ of PBS solution of the MTT tetrazolium substrate $(5 \mathrm{mg} / \mathrm{ml})$ was added after 1, 4 and 7 days. After incuba- tion for $4 \mathrm{~h}$ at $37^{\circ} \mathrm{C}$, the resulting purple formazan precipitate was solubilized by the addition of $1 \mathrm{ml}$ of DMSO and shaken for $30 \mathrm{~min}$. An aliquot from each well $(100 \mu \mathrm{l})$ was transferred to 96 -well plates and then read using a plate reader of an ELISA (E-max, Molecular Device, USA). The optical density of each well determined at $590 \mathrm{~nm}$.

\section{RESULTS AND DISCUSSION}

\subsection{Preparation of PTx-Loaded SMES}

PLGA of different molecular weights used to compare the stability of the self-microemulsifying PTx. To prepare self-microemulsifying PTx consisting of mixtures of Ptx, oil, a surfactant and PLGA, the formulation is summarized in Table 1. PLGA and PTx were dissolved by solubilizers which were a mixture of tetraglycol, Cremophor ELP, and Labrasol.

Firstly, the prepared PTx-loaded SMES were observed visually. As shown in Figure 1(a), PTx-loaded SMES (F1-0 - F10-0) showed the emulsion solution from transparent to semitransparent according to various formulations. The droplet size for all formulations of PTx-loaded SMES was found in the range of $80-130$ $\mathrm{nm}$. The droplet size distribution is comparatively narrow for all formulations.

The morphology of PTx-loaded SMES was measured by AFM as shown in Figure 2. PTx-loaded SMES (F10) showed the spherical shape with smooth surface. A comparatively uniform droplet size of PTx-loaded SMES was also observed at AFM, indicating no aggregation or adhesion among SMES.

\subsection{Stability of PTx-Loaded SMES}

The storage stability of PTx-loaded SMES is important to maintain the therapeutic concentration of PTx. Therefore, PTx-loaded SMES was firstly prepared with DW or a solution of $0.9 \% \mathrm{NaCl}$ and $5 \%$ bovine serum albumin. The prepared PTx-loaded SMES was constantly shaken at $100 \mathrm{rpm}$ and $37^{\circ} \mathrm{C}$ for 7 days to examine the storage stability. After 4 and 7 days, there is no change of particle size for F1-F5 as shown in Figure 1(b), indicating the storage stability of PTx-loaded SMES in DW. Meanwhile, F6 and F7 with PTx content $<0.05$ wt showed the precipitation of PTx. Hence we investigated the SMES with PTx content of $0.003 \mathrm{wt}$ according to different molecular weights of PLGA (F8-F10). F8, F9 and F10 in DW showed no change of particle size at $37^{\circ} \mathrm{C}$ for 4 and 7 days (Table 2).

Next, PTx-loaded SMES was prepared with a solution of $0.9 \% \mathrm{NaCl}$ and $5 \%$ bovine serum albumin as a model blood condition. The haze color of PTx-loaded SMES is deeply changed according to increasing incubation time (Figure 3). The particle size of F8 and F10 increased to 
Table 1. Formulation for the preparation of PTx-loaded SMES.

\begin{tabular}{|c|c|c|c|c|c|c|c|c|c|c|}
\hline \multirow{2}{*}{ Composition $(\mathrm{g}, \mathrm{w} / \mathrm{w})$} & \multicolumn{10}{|c|}{ Formulation } \\
\hline & F1 & F2 & F3 & F4 & F5 & F6 & F7 & F8 & F9 & F10 \\
\hline Drug (Paclitaxel) & & 0.001 & & 0.001 & 0.003 & 0.05 & 0.01 & & 0.003 & \\
\hline Solubilizer (Tetraglycol) & & 0.5 & & & & & & & 0.5 & \\
\hline PLGA 8 kg/mol & 0.005 & 0.01 & 0.015 & & & & & 0.005 & & \\
\hline PLGA 20 kg/mol & & & & & & & & & 0.005 & \\
\hline PLGA 90 kg/mol & & & & & & & & & & 0.005 \\
\hline Cosurfactant (Labrasol) & & 0.14 & & & & & & & 0.14 & \\
\hline Surfactant (Cremophor ELP) & & 0.16 & & & & & & & 0.16 & \\
\hline
\end{tabular}
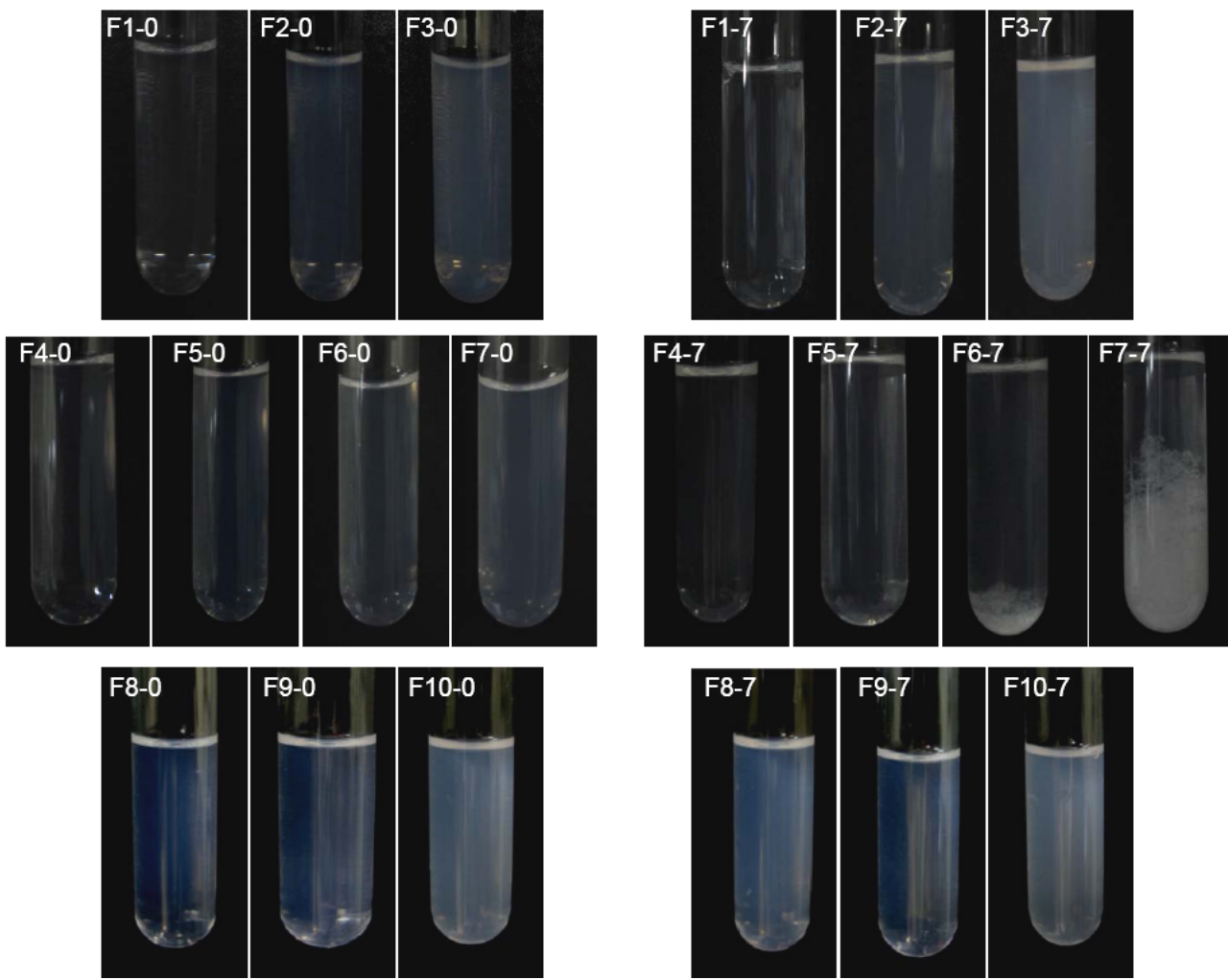

(a)

(b)

Figure 1. Pictures (a) before and (b) after incubation at $37^{\circ} \mathrm{C}$ for 7 days of PTx-loaded SMES prepared in DW with different formulations F1-F10.

Table 2. The changes of particle size by incubation for $0-7$ days at $37^{\circ} \mathrm{C}$ of PTx-loaded SMES prepared in DW and blood condition with different formulations F8-F10.

\begin{tabular}{|c|c|c|c|c|c|c|c|}
\hline \multicolumn{2}{|c|}{ Condition } & \multicolumn{3}{|c|}{ In DW } & \multicolumn{3}{|c|}{ In blood condition ${ }^{a}$} \\
\hline Form & & F8 & F9 & F10 & F8 & F9 & F10 \\
\hline \multirow{3}{*}{$\begin{array}{l}\text { Particle size } \\
(\mathrm{nm})^{\mathrm{b}}\end{array}$} & Initial & $80 \pm 1$ & $81 \pm 1$ & $128 \pm 3$ & $1483 \pm 219$ & $70 \pm 1$ & $162 \pm 4$ \\
\hline & 4 days & $84 \pm 3$ & $85 \pm 3$ & $129 \pm 4$ & $1920 \pm 518$ & $301 \pm 152$ & $1399 \pm 791$ \\
\hline & 7 days & $80 \pm 2$ & $104 \pm 2$ & $124 \pm 1$ & $1970 \pm 709$ & $773 \pm 288$ & $1005 \pm 140$ \\
\hline
\end{tabular}

${ }^{\mathrm{a}}$ A solution of $0.9 \% \mathrm{NaCl}$ and $5 \%$ bovine serum albumin; ${ }^{\mathrm{b}}$ The mean and standard deviation of particle size for each formulation was calculated by individual measurement of three formulations. 

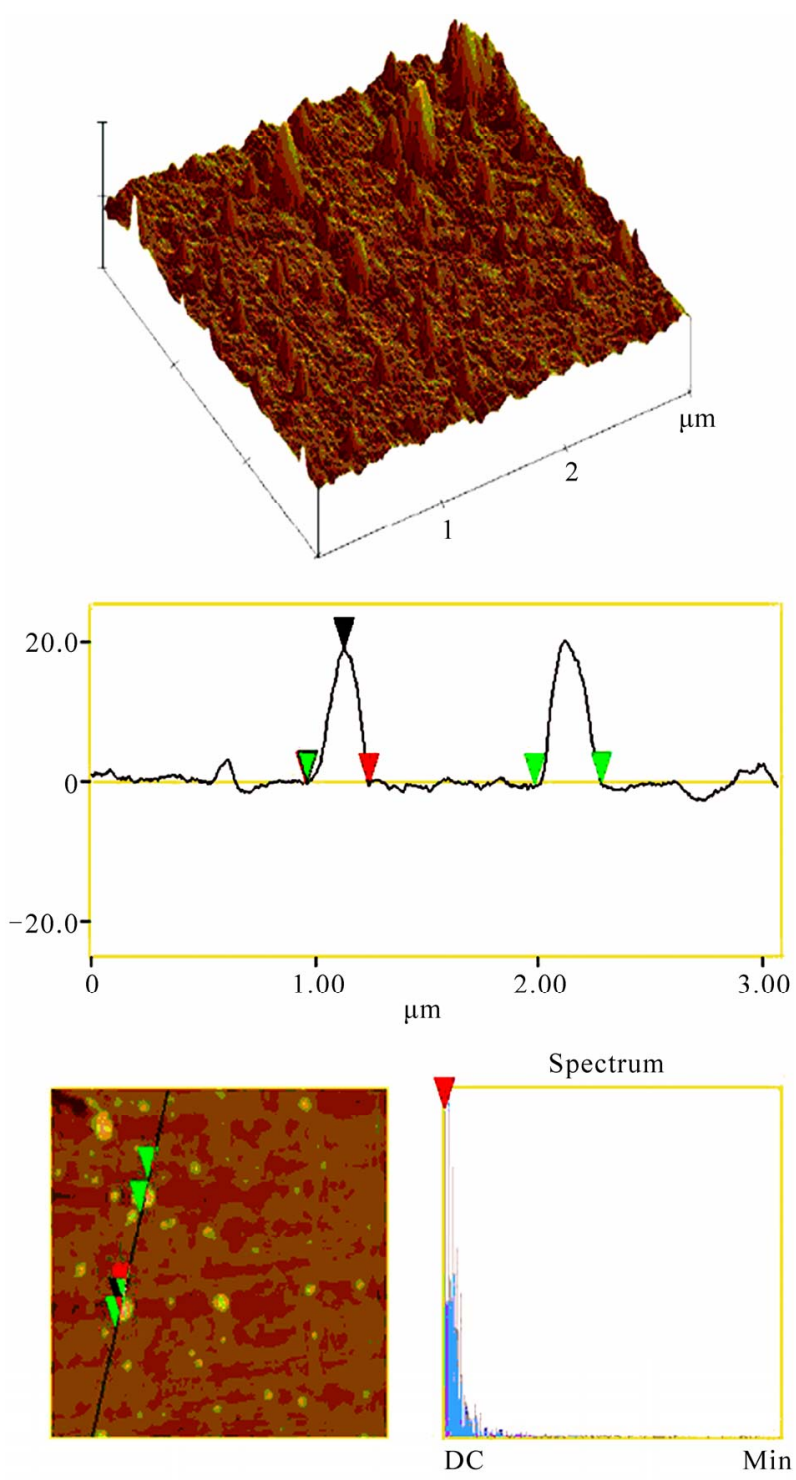

Figure 2. AFM image of PTx-loaded SMES (F10).
1000 - $2000 \mathrm{~nm}$ after incubation, while that of formulation F9 increased to $800 \mathrm{~nm}$ (Table 2). Even though these formulations showed the increasing in particle size, there is no precipitation under even blood condition. This study does not provide sufficient data to allow acomplete description of the stability to be proposed, however, it indicated that the stability of PTx-loaded SMES depended on the molecular weights of PLGA and concentration of PTx.

\subsection{Anti-Tumor Activity of PTx-Loaded SMES}

The formulations F8-F10 were examined for their antiproliferative activities against B16F10 melanoma cell line (Figure 4). The population of B16F10 cells shapely increased as a function of culture time after addition of saline (control). The PTx-loaded SMES (F8-F10) exerted a significant inhibitory effect on cell proliferation. The cell viability was approximately $40 \%$ and $20 \%$ at 4 days and 7 days, respectively. This indicated that PTXloaded SMES (F8-F10) displayed marked inhibition of B16F10 cell proliferation.

\subsection{Preparation of PTx-Loaded SMES}

PLGA of different molecular weights used to compare the stability of the self-microemulsifying PTx. To prepare self-microemulsifying PTx consisting of mixtures of Ptx, oil, a surfactant and PLGA, the formulation is summarized in Table 1. PLGA and PTx were dissolved by solubilizers which were a mixture of tetraglycol, Cremophor ELP, and Labrasol.

Firstly, the prepared PTx-loaded SMES were observed visually. As shown in Figure 1(a), PTx-loaded SMES (F1-0 - F10-0) showed the emulsion solution from transparent to semitransparent according to various formulations. The droplet size for all formulations of PTX-loaded SMES was found in the range of $80-130 \mathrm{~nm}$.

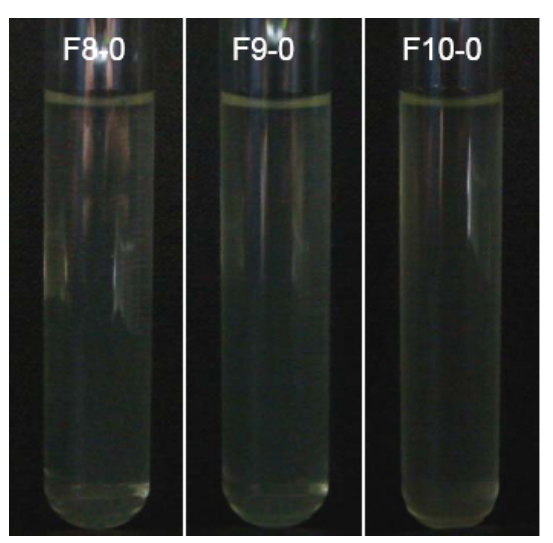

(a)

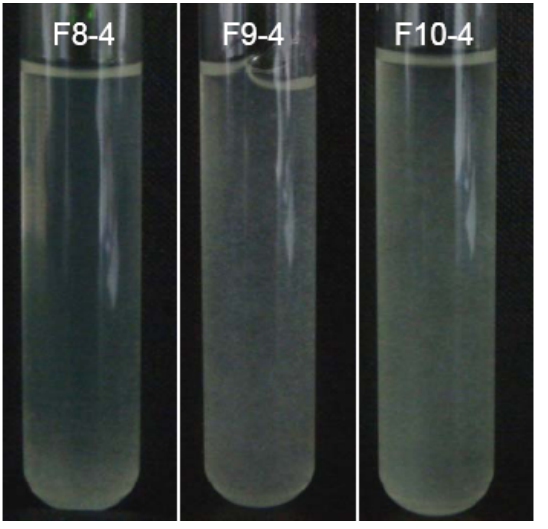

(b)

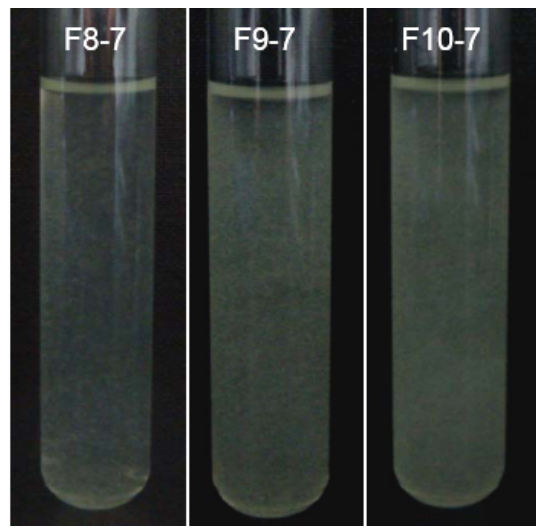

(c)

Figure 3. Pictures (a) before and after incubation for (b) 4 days and (c) 7 days at $37^{\circ} \mathrm{C}$ of PTx-loaded SMES prepared in $5 \%$ BSA with different formulations F8-F10. 


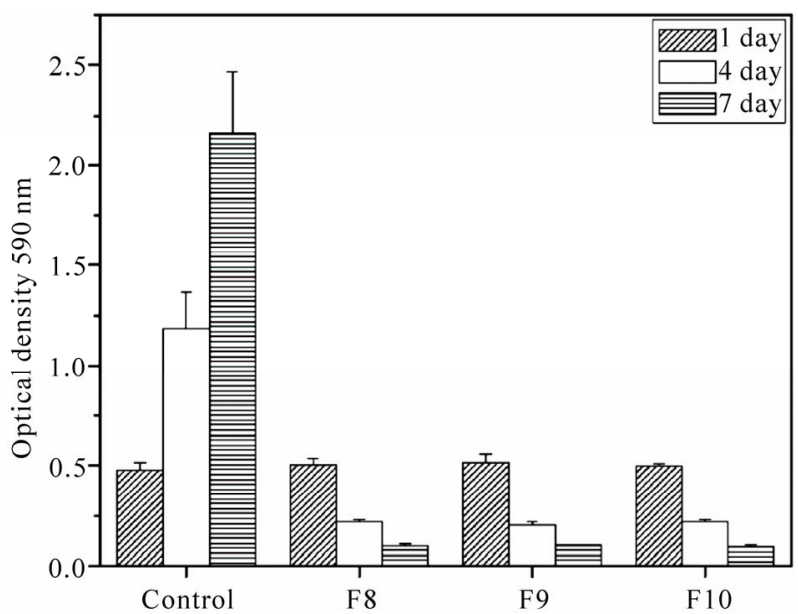

Figure 4. In vitro cytotoxicity of B16F10 melanoma cell against PTx-loaded SMES of formulations F8, F9 and F10 for 1,4 and 7 days. The cells grown on a culture plate without PTx-loaded SMES were used as the control.

The droplet size distribution is comparatively narrow for all formulations.

The morphology of PTx-loaded SMES was measured by AFM as shown in Figure 2. PTx-loaded SMES (F10) showed the spherical shape with smooth surface. A comparatively uniform droplet size of PTx-loaded SMES was also observed at AFM, indicating no aggregation or adhesion among SMES.

\section{CONCLUSIONS}

We prepared the PTx-loaded SMES with different formulations to examine the storage stability. The prepared PTx-loaded SMES showed a spherical shape with ranging of $100 \mathrm{~nm}$. We found the formulation of the PTxloaded SMES with stability for 7 days. The formulation in this work could be used as administration form for animal trials. Thus, further research on the animal model using PTx-loaded SMES prepared in this work is now in progress.

\section{ACKNOWLEDGEMENTS}

This work was supported by a grant from the new faculty research fund of Ajou University, KMOHW (grant no A050082) and Priority Research Centers Program through NRF funded by the Ministry of Education, Science and Technology (2010-0028294).

\section{REFERENCES}

[1] Li, J.Y., Strobel, G., Sidhu, R., Hess, W.M. and Ford, E.J. (1996) Endophytic taxol-producing fungi from bald cypress, Taxodium distichum. Microbiology, 142, 22232226. doi:10.1099/13500872-142-8-2223

[2] Spencer, C.M. and Faulds, D. (1994) Paclitaxel: A review of its pharmacodynamic and pharmacokinetic properties and therapeutic potential in the treatment of cancer. Drugs, 48, 794-847.

[3] Scripture, C.D., Szebeni, J., Loos, W.J., Figg, W.D. and Sparreboom, A. (2005) Comparative in vitro properties and clinical pharmacokinetics of Paclitaxel following the administration of taxol(r) and paxene(r). Cancer Biology \& Therapy, 4, 555-560.

[4] Cordes, N. and Plasswilm, L. (1998) Cell line and schedule-dependent cytotoxicity of paclitaxel (Taxol): Role of the solvent Cremophor EL/ethanol. Anticancer Research, 18, 1851-1857.

[5] Pang, K.S., Maeng, H.J. and Fan, J. (2009) Interplay of transporters and enzymes in drug and metabolite processing. Molecular Pharmaceutics, 6, 1734-1755. doi:10.1021/mp900258z

[6] Zhang, C., Qu, G., Sun, Y., Wu, X., Yao, Z., Guo, Q., Ding, Q., Yuan, S., Shen, Z., Ping, Q. and Zhou, H. (2008) Pharmacokinetics, biodistribution, efficacy and safety of N-octyl-O-sulfate chitosan micelles loaded with paclitaxel. Biomaterials, 29, 1233-1241. doi:10.1016/j.biomaterials.2007.11.029

[7] Wei, H., Cheng, S.X., Zhang, X.Z. and Zhuo, R.X. (2009) Thermo-sensitive polymeric micelles based on poly (N-isopropylacrylamide) as drug carriers. Progress in Polymer Science, 34, 893-910. doi:10.1016/j.progpolymsci.2009.05.002

[8] Lee, H.B., Jeong, J.K., Sohn, S.I., Byun, Y., Ki, M.H. and Seo, J.K. (2009) Drug delivery and regenerative medicine technology. Tissue Engineering Regenerative Medicine, 4, 663-667.

[9] Yu, W. and Zhang, N. (2009) Surface modification of nanocarriers for cancer therapy. Current Nanoscience, 5, 123-134. doi:10.2174/157341309788185370

[10] Feng, S. and Huang, G. (2001) Effects of emulsifiers on the controlled release of paclitaxel (Taxol) from nanospheres of biodegradable polymers. Journal of Controlled Release, 71, 53-69. doi:10.1016/S0168-3659(00)00364-3

[11] Gao, P., Rush, B.D., Pfund, W.P., Huang, T., Bauer, J.M., Morozowich, W., Kuo, M.S. and Hageman, M.J. (2003) Development of a supersaturable SEDDS (S-SEDDS) formulation of paclitaxel with improved oral bioavailability. Journal of Pharmaceutical Sciences, 92, 23862398. doi:10.1002/jps.10511

[12] Gursoy, N., Garrigue, J.S., Razafindratsita, A., Lambert, G. and Benita, S. (2003) Excipient effects on in vitro cytotoxicity of a novel paclitaxel self-emulsifying drug delivery system. Journal of Pharmaceutical Sciences, 92 2411-2418. doi:10.1002/jps.10501

[13] Ten Tije, A.J., Verweij, J., Loos, W.J. and Sparreboom, A (2003) Pharmacological effects of formulation vehicles: implications for cancer chemotherapy. Clinical Pharmacokinetics, 42, 665-685.

[14] Kan, P., Chen, Z.B., Lee, C.J. and Chu, I.M. (1999) Development of nonionic surfactant/phospholipid o/w emulsion as a paclitaxel delivery system. Journal of Controlled Release, 58, 271-278. doi:10.1016/S0168-3659(98)00164-3 